\title{
WHEN PROPER MAPS ARE CLOSED ${ }^{1}$
}

\author{
RICHARD S. PALAIS
}

A subset $F$ of a space $Y$ is called compactly closed if its intersection with each compact subset of $Y$ is compact. A Hausdorff space is called a $k$-space if every compactly closed set is closed. A map of a space $X$ to a space $Y$ is called proper if the inverse image of each compact subset of $Y$ is a compact subset of $X$. The following Lemma is a trivial consequence of these definitions. ${ }^{2}$

Lemma. A subset $F$ of a space $Y$ is compactly closed if and only if the inclusion map of the subspace $F$ into $Y$ is proper.

It is frequently of interest in applications to know that a given proper map is closed. We provide here a simple proof of a criterion due to G. T. Whyburn [1]. ${ }^{3}$

THEOREM. The following four properties of a space $Y$ are equivalent:

(a) Every surjective proper map $g: Z \rightarrow Y$ is closed.

(b) Every proper map $f: X \rightarrow Y$ is closed.

(c) Every injective proper map $h: X \rightarrow Y$ is closed.

(d) Every compactly closed subset $F$ of $Y$ is closed.

Proof. Assuming (a) and given a proper $\operatorname{map} f: X \rightarrow Y$, let $Z$ denote the topological sum of $X$ and $Y$ and define $g: Z \rightarrow Y$ by $g \mid X=f$ and $g \mid Y=i_{Y}$. The $g$ is clearly surjective and proper, hence closed, and since $X$ is closed in $Z, f$ is closed. Hence (a) $\Rightarrow(b)$. Trivially (b) $\Rightarrow(c)$, while $(c) \Rightarrow(d)$ is immediate from the Lemma. Since $(b) \Rightarrow(a)$ is trivial it remains to deduce (b) from (d). Let $f: X \rightarrow Y$ be a proper map and $F$ a closed subset of $X$. We must show $f(F)$ is closed in $Y$, and since we are assuming (d), it suffices to show that if $K$ is a compact subset of $Y$ then $K \cap f(F)$ is compact. But $K \cap f(F)=f\left(f^{-1}(K) \cap F\right)$.

Received by the editors June 1, 1969.

1 Research supported in part by Air Force Grant AFOSR 68-1403.

2 The editor suggests we caution the reader that the usage of these terms in the literature is not uniform and each has been used elsewhere with at least one other meaning.

${ }^{3}$ I would like to thank E. Michael for pointing out to me Whyburn's earlier proof and also the implication $(\mathrm{a}) \Rightarrow(\mathrm{b})$. 
Since $f$ is proper, $f^{-1}(K)$ and hence $f^{-1}(K) \cap F$ are compact and hence so is $f\left(f^{-1}(K) \cap F\right)$. Q.E.D.

It is well known and trivial that a Hausdorff space satisfying the first axiom of countability is a $k$-space, hence:

Corollary. If $Y$ is a metrizable space or, more generally, a firstcountable Hausdorff space or, more generally still, a $k$-space then every proper map into $Y$ is closed.

\section{REFERENCE}

1. G. T. Whyburn, Directed families of sets and closedness of functions, Proc. Nat. Acad. Sci. U.S.A. 54 (1965), 688-692. MR 32 \#335.

BRANDEIS UNIVERSITY AND

The Institute for Advanced Study 